\title{
Propiedades tribológicas de los materiales compuestos de matriz de aluminio reforzados con partículas cerámicas
}

\author{
A. Forn-Alonso*, M.T. Baile-Puig* y E. Rupérez-de-Gracia*
}

\begin{abstract}
Resumen En el presente trabajo se estudia el comportamiento frente al desgaste de la aleación de aluminio 6061 reforzada con partículas de $\mathrm{Al}_{2} \mathrm{O}_{3}$, con un tamaño medio de partícula de 13,6 $\mu \mathrm{m}$. El material, obtenido mediante proceso "Duralcan", ha sido extruido en caliente y, posteriormente, sometido a un tratamiento térmico T6. Utilizando un equipo de Pin-ondisc, con pin esférico de WC-6\% Co, se han evaluado la resistencia al desgaste y el coeficiente de fricción para diferentes condiciones de carga, a temperatura ambiente y en ausencia de lubricación. También se ha estudiado la evolución del desgaste producido a distinto número de ciclos. El análisis de la huella de desgaste y de la sección perpendicular a la superficie desgastada, mediante microscopía eléctronica de barrido (SEM), ha permitido conocer el mecanismo asociado al desgaste en las diferentes condiciones ensayadas, encontrándose, en todos los casos, desgaste por abrasión.
\end{abstract}

Palabras clave Materiales compuestos. Aluminio. Desgaste. Fricción.

\section{Wear behaviour of aluminium metal matrix composites reinforced with ceramic particles}

\begin{abstract}
The wear behaviour of the reinforced aluminium alloy $6061 /\left(\mathrm{Al}_{2} \mathrm{O}_{3}\right) \mathrm{p}$, with an average particle size of $13,6 \mu \mathrm{m}$, is studied. The material, obtained through process Duralcan, has been hot extruded and after submitted to thermal treatment T6. Using a "Pin-on-disc" equipment with WC- $6 \%$ Co spherical pin, the resistance to the wear and the friction coefficient, in different load conditions, at room temperature under dry conditions have been evaluated. The wear rate as a distance covered has also been evaluated. The analysis of a perpendicular section to the worn surface, through scanning electronic microscopy, has permitted to know the wear associated mechanism in different tested conditions. In all cases, the main wear mechanism involved was abrasive wear.
\end{abstract}

Keywords Composites. Aluminium. Wear. Friction.

\section{INTRODUCCIÓN}

Los materiales compuestos de matriz metálica (MMCs) son utilizados industrialmente debido a sus excelentes propiedades mecánicas y su resistencia al desgaste. El estudio del comportamiento al desgaste de los materiales $\mathrm{MMC}$ es interesante porque aleaciones relativamente blandas, como las de aluminio, pueden mejorar significativamente su comportamiento al desgaste, introduciendo partículas de materiales cerámicos como son el óxido de aluminio $\left(\mathrm{Al}_{2} \mathrm{O}_{3}\right)$ y el carburo de silicio $(\mathrm{SiC})$. $\mathrm{La}$ resistencia de refuerzos discontinuos en los MMCs no es tan elevada como la de materiales compuestos con refuerzo continuo, pero las propiedades y el cos- te de los MMCs discontinuos hace que sean útiles como materiales resistentes al desgaste.

Algunos estudios muestran que la resistencia al desgaste de los materiales compuestos de matriz de aluminio disminuye con la fracción en volumen del refuerzo y, drásticamente, con el grado de porosidad del material si la comparamos con la correspondiente aleación sin refuerzo ${ }^{[1]}$. Esto, puede ser debido a los defectos presentes en el material compuesto.

El comportamiento de los materiales en sistemas tribológicos depende, principalmente, del tipo de contacto, de los niveles de tensiones mecánicas aplicadas, de la velocidad relativa entre los dos materiales en contacto, de la temperatura, de la humedad, de la naturaleza del entorno y de la geometría

(*) Centro de Diseño de Aleaciones Ligeras y Tratamientos de Superficie (CDAL). Universitat Politècnica de Catalunya. 
del sistema ${ }^{[2}$ y 3$]$. Por tanto, el desgaste es esencialmente una propiedad del sistema estudiado.

En este trabajo se ha analizado la influencia de la carga aplicada, en el comportamiento al desgaste de materiales compuestos base aluminio con partículas cerámicas de refuerzo, así como la evolución de dicho desgaste con la distancia recorrida.

\section{MATERIALES Y PROCEDIMIENTO EXPERIMEN- TAL}

\subsection{Material}

El material objeto de esta investigación es la aleación Al-6061 reforzado con un $26 \%$ de partículas de $\mathrm{Al}_{2} \mathrm{O}_{3}$, con un tamaño medio de $13,6 \mu \mathrm{m}$ y un factor de forma, definido como anchura/longitud, de 0,58. El material ha sido suministrado por "Duralcan" en forma de lingotes de $270 \mathrm{~mm}$ de diámetro y ha sido obtenido mediante un proceso de colada.

La composición de la matriz de aluminio 6061 MMC se ha determinado por espectroscopía óptica de emisión (OES) y está indicada en la tabla I.

Las partículas de refuerzo están formadas por polvo de óxido de aluminio calcinado, con una composición química del 98,5\% de $\mathrm{Al}_{2} \mathrm{O}_{3}$.

El lingote ha sido extruido a una temperatura de $450^{\circ} \mathrm{C}$, para obtener barras de $88,9 \mathrm{~mm}$ de diámetro.

Muestras del material compuesto extruido han sido sometidas a un tratamiento térmico T6: puesta en solución a $560{ }^{\circ} \mathrm{C}$ durante $2 \mathrm{~h}$, temple en agua a $25^{\circ} \mathrm{C}$ y envejecimiento artificial a $175^{\circ} \mathrm{C}$ durante $10 \mathrm{~h}$.

\subsection{Procedimiento experimental}

Para conocer las propiedades tribológicas se han realizado ensayos de fricción con un equipo pin-ondisk CSEM, según la norma ASTM G99-95a, que dispone de un sistema de control de humedad y temperatura.

Los ensayos se llevaron a cabo sin lubricación, a temperatura ambiente y a una humedad relativa del $50 \%$. Se ha utilizado como pin una bola de WC- $6 \%$ Co de $6 \mathrm{~mm}$ de diámetro, con una rugosidad Ra de 0,02 $\mu \mathrm{m}$, una dureza de $1.600 \mathrm{HV}_{0,3}$, un módulo de Young de 630 GPa y un coeficiente de Poisson de 0,21.

Los discos, de $50 \mathrm{~mm}$ de diámetro y $10 \mathrm{~mm}$ de espesor, se mecanizaron a partir del material compuesto extruido, y posteriormente fueron tratados térmicamente (T6) y desbastados hasta obtener una rugosidad superficial de $0,15 \mu \mathrm{m}$.

Los ensayos se han realizado a una velocidad lineal de $10 \mathrm{~cm} / \mathrm{s}$ y aplicando cargas de $10,20,30$ y 40 N. En todos los casos, la distancia de deslizamiento ha sido de 20.000 ciclos.

Se ha utilizado un rugosímetro-perfilómetro TAYLOR-HOBSON para medir las rugosidades de los discos y para evaluar el desgaste producido, mediante la medida de la profundidad y anchura la huella de desgaste.

Posteriormente a los ensayos de fricción, y con la finalidad de conocer el tipo y el mecanismo de desgaste, se estudiaron tanto las huellas de desgaste como la sección transversal de la superficie desgastada, mediante un microscopio electrónico de barrido JEOL JSM-5600.

\section{RESULTADOS Y DISCUSIÓN}

Los resultados de desgaste para diferentes cargas, a una velocidad constante de $10 \mathrm{~cm} / \mathrm{s}$, están indicados en la tabla II.

Tabla II. Desgaste del material $6061 / /\left(\mathrm{Al}_{2} \mathrm{O}_{3}\right)_{p}$ a diferentes cargas.

Table II. $6061 / /\left(\mathrm{Al}_{2} \mathrm{O}_{3}\right)_{p}$ wear behaviour to different loads

\begin{tabular}{lcccc}
\hline $\begin{array}{l}\text { Carga } \\
(\mathrm{N})\end{array}$ & $\begin{array}{c}\text { Coef. } \\
\text { Fricción }\end{array}$ & $\begin{array}{c}\text { Desgaste } \\
\left(\mathrm{m}^{2} / \mathrm{N}\right)\end{array}$ & $\begin{array}{c}\text { Velocidad de } \\
\text { desgaste } \\
\left(\mathrm{mm}^{3} / \mathrm{m}\right)\end{array}$ & $\begin{array}{c}\text { Pérdida de } \\
\text { peso } \\
(\mathrm{mg} / \mathrm{m})\end{array}$ \\
\hline 10 & 0,47 & $2,3010^{-13}$ & $2,1010^{-3}$ & $1,5210^{-3}$ \\
20 & 0,36 & $2,0810^{-13}$ & $3,2710^{-3}$ & $7,2810^{-3}$ \\
30 & 0,31 & $1,5610^{-13}$ & $4,3310^{-3}$ & $1,0710^{-2}$ \\
40 & 0,26 & $1,4310^{-13}$ & $4,7810^{-3}$ & $1,1610^{-2}$ \\
\hline
\end{tabular}

Tabla I. Composición química de la aleación de aluminio 6061 por OES (\% en masa)

Table I. Chemical composition of 6061 aluminium alloy by OES (mass \%)

\begin{tabular}{lcccccccccc}
\hline $\mathrm{Si}$ & $\mathrm{Mg}$ & $\mathrm{Cu}$ & $\mathrm{Ni}$ & $\mathrm{Ti}$ & $\mathrm{Cr}$ & $\mathrm{Fe}$ & $\mathrm{Zn}$ & $\mathrm{Mn}$ & $\mathrm{Al}$ \\
\hline 0,56 & 0,99 & 0,23 & 0,001 & 0,007 & 0,12 & 0,12 & 0,003 & 0,004 & resto \\
\hline
\end{tabular}


Propiedades tribológicas de los materiales compuestos base aluminio reforzados con partículas cerámicas A. Forn-Alonso, M.T. BAile-Puig y E. Rupérez-DE Gracia

Se ha encontrado que tanto el coeficiente de fricción $(\mu)$ como el coeficiente de desgaste disminuyen al aumentar la carga aplicada (Figs. 1 y 2 ).

Mediante microscopía electrónica de barrido (SEM) se han analizado las huellas de desgaste producidos a distintas cargas. Las mismas se muestran en la figura 3.

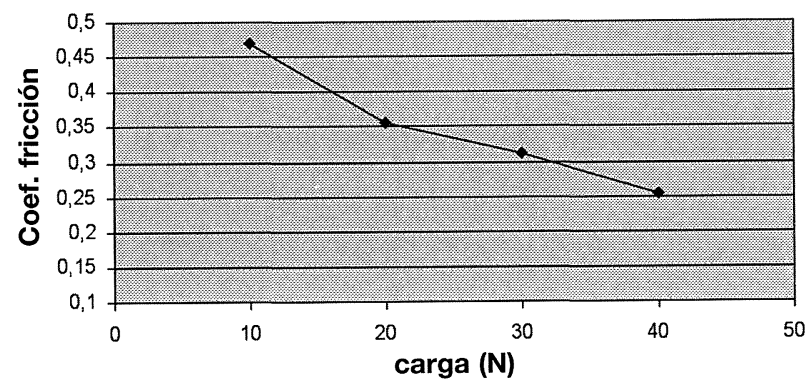

Figura 1. Variación del coeficiente de fricción del material 6061 MMC en función de la carga.

Figure 1. Friction coefficient variation according to the load in the 6061 MMC material.
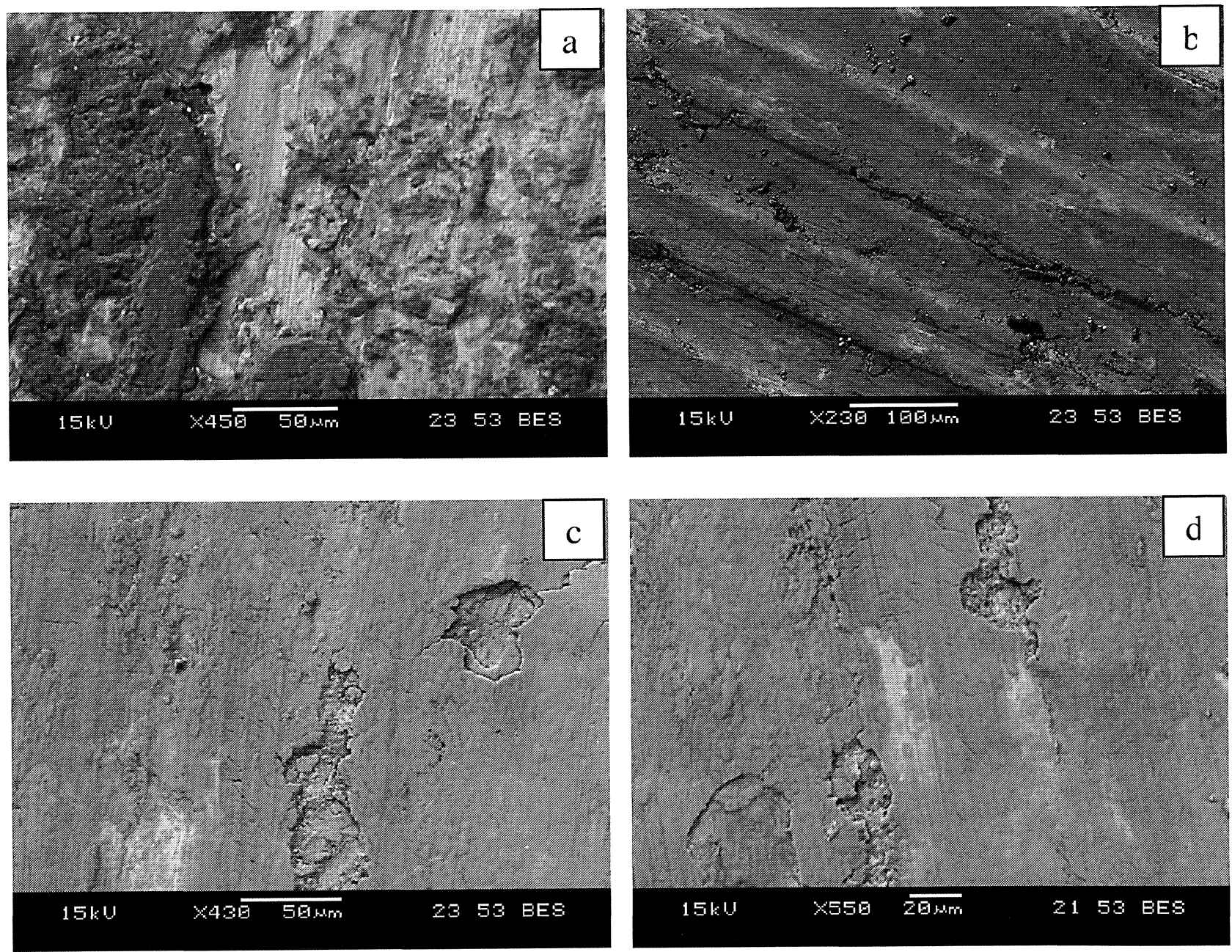

Figura 3. Micrografía SEM de las superficies desgastadas para cargas de: a) $10 \mathrm{~N}$, b) $20 \mathrm{~N}, \mathrm{c}) 30 \mathrm{~N}$ y d) $40 \mathrm{~N}$.

Figure 3. SEM micrograph of worn surfaces for loads: a) $10 \mathrm{~N}$, b/20N, c) $30 \mathrm{~N}$ y d) $40 \mathrm{~N}$.

Rev. Metal. Madrid Vol. Extr. (2005) 193-196 
Propiedades tribológicas de los materiales compuestos base aluminio reforzados con partículas cerámicas A. Forn-Alonso, M.T. BAILE-PUIG y E. RuPÉREz-DE GRACIA

partículas arrancadas quedan depositadas sobre la huella de desgaste, principalmente en los laterales de la misma.

Un análisis de la sección perpendicular a la huella de desgaste (Fig. 4) muestra que una carga de $10 \mathrm{~N}$ es suficiente para producir la fractura de las partículas de refuerzo, siendo la zona afectada de $5-10 \mu \mathrm{m}$ por debajo de la superficie desgastada (Fig. 4a) frente a las $15-20 \mu \mathrm{m}$ en el caso de cargas de $20 \mathrm{~N}$ (Fig. 4b).

También se ha evaluado el comportamiento al desgaste, a distinto número de ciclos bajo una misma carga. En la gráfica de la figura 5 se observa una disminución brusca del coeficiente de desgaste hasta 1.000 ciclos y, posteriormente, se estabiliza a un valor aproximado de $1,5 \cdot 10^{-13} \mathrm{~m}^{2} / \mathrm{N}$. Tal y como han sugerido otros autores ${ }^{[56]}$ podemos hablar de una transición desde un desgaste severo por debajo de 1.000 ciclos a un desgaste suave por encima de los 1.000 ciclos.
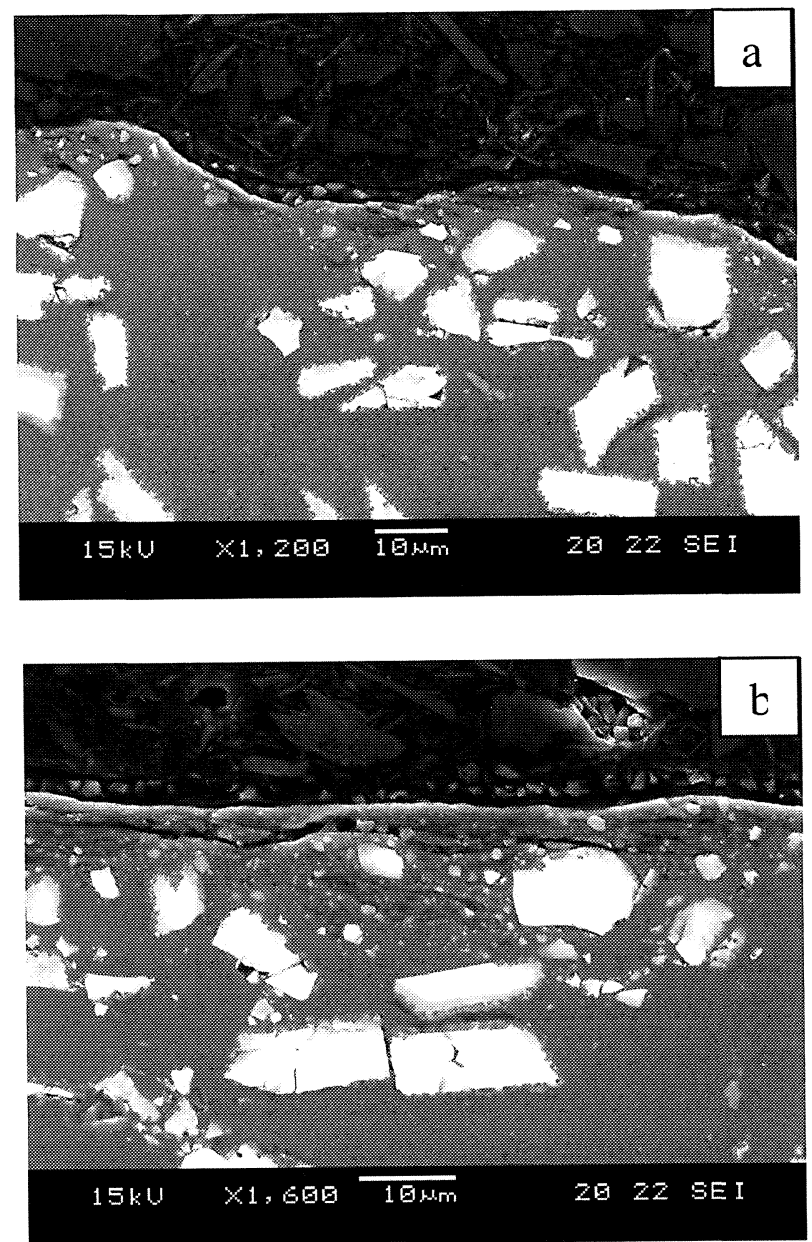

Figura 4. Sección tranversal de la superficie desgastada bajo una carga de: a) $10 \mathrm{~N}$ y b) $20 \mathrm{~N}$.

Figure 4. Tranversal section of worn surfaces under a load of: a) $10 \mathrm{~N}$ y b/20N.

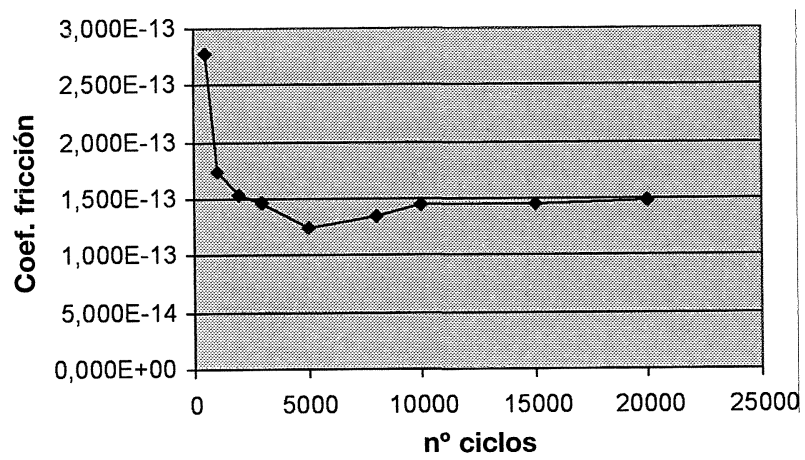

Figura 5. Evolución del desgaste del material $6061 \mathrm{MMC}$ a diferente número de ciclos.

Figure 5. $6061 \mathrm{MMC}$ material wear behaviour for different cycle numbers.

\section{CONCLUSIONES}

- El desgaste es abrasivo para todas las cargas ensayadas.

- La rugosidad de la huella de desgaste disminuye al aumentar la carga aplicada.

- El coeficiente de fricción disminuye al aumentar la carga aplicada: Sus valores oscilan entre 0,50 y 0,25 para cargas de 10 y $40 \mathrm{~N}$ respectivamente.

- El coeficiente de desgaste disminuye al aumentar el número de ciclos y se observa una transición a los 1000 ciclos, pasando de un desgaste severo a uno más suave.

\section{Agradecimientos}

Los autores desean agradecer al Ministerio de Ciencia y Tecnología la ayuda recibida a través de los proyectos CICYT MAT2001-1732-C02-01/02 y DPI2002-04581-C02-01.

\section{REFERENCIAS}

[1] O. Yilmaz y S. Buytoz, Compos. Sci. Technol. 61 (2001) 2.381-2.392.

[2] A. Martin, M.A. Martinez y J. Llorca, An. Mecánica de la Fractura 12 (1995) 284-289.

[3] B.K. YeN, J. Mater. Sci. 32 (1997) 821-828.

[4] E. Martín, A. Forn y J.A. PiCAs, $8^{\circ}$ Congreso Nacional de Propiedades Mecánicas de Sólidos, 2002, 867-874.

[5] L.J. YanG, Compos. Sci. Technol. 63 (2003) 9. Article in press.

[6] S.J. Lin, G.A. Wu y J.L. Horng, J. Mater. Sci. 31 (1996) 